\title{
NUEVAS ESPECIES DE LA FAMILIA MECYSMAUCHENIIDAE (ARANEAE) DE CHILE Y ARGENTINA.
}

\author{
Cristian J. Grismado ${ }^{1}$ \& Martín J. Ramírez ${ }^{1}$
}

Biota Neotropica v5 (n1a) - http://www.biotaneotropica.org.br/v5n1a/pt/abstract?taxonomic-review+bn019051a2005

Recebido em 07/12/2003

Publicado em 01/02/2005

División Aracnología, Museo Argentino de Ciencias Naturales “Bernardino Rivadavia”, Av. Angel Gallardo 470 C1405DJR, Buenos Aires, Argentina. E-mail: grismado@macn.gov.ar, ramirez@macn.gov.ar

\begin{abstract}
Two new species of the family Mecysmaucheniidae are described: Mecysmauchenius platnicki n. sp., from Malleco, Chile and Mecysmauchenioides quetrihue n. sp., from Neuquén, Argentina, the latter being the second known species of the genus.
\end{abstract}

Key words: Araneae, Mecysmaucheniidae, Mecysmauchenius, Mecysmauchenioides, new species.

\section{Resumen}

Se describen dos nuevas especies de la familia Mecysmaucheniidae: Mecysmauchenius platnicki, sp. nov., de Malleco, Chile y Mecysmauchenioides quetrihue, sp. nov., de Neuquén, Argentina, constituyendo esta última la segunda especie conocida de su género.

Palabras-clave: Araneae, Mecysmaucheniidae, Mecysmauchenius, Mecysmauchenioides, nuevas especies. 


\section{INTRODUCCIÓN}

Entre los grupos faunísticos característicos de la zona austral de América del Sur -la cual se destaca porque gran parte de su flora y fauna presenta más afinidades con las de Australasia y Sudáfrica que con el resto de Sudamérica (Cabrera \& Willink, 1973; Morrone, 1999, 2000)- se encuentra la familia de arañas Mecysmaucheniidae. Comprende curiosas arañas criptozoicas caracterizadas esencialmente por poseer sólo dos hileras y quelíceros enormemente alargados que surgen de una especie de foramen formado por los márgenes laterales del cefalotórax al unirse ventralmente (Forster y Platnick, 1984). Las Mecysmaucheniidae habitan el sur de Sudamérica y Nueva Zelanda. En el nuevo mundo se conocen cinco géneros; Mecysmauchenius Simon, 1884 es el más especioso (dieciséis especies descriptas), y los restantes cuatro, descriptos por Forster y Platnick (1984), son monotípicos (Mecysmauchenioides, Semysmauchenius, Mesarchaea y Chilarchaea). En Nueva Zelanda se conocen dos géneros, Aotearoa Forster y Platnick, 1984 y Zearchaea Wilton, 1946. Se las ha colectado principalmente en el mantillo de los húmedos bosques australes y se sabe que -al menos algunas de las especies- son araneófagas (Forster y Platnick, 1984; Grismado y Lopardo, 2003). También resulta notorio el hecho de que en ciertas localidades, varias especies viven en simpatría (Grismado y Lopardo, 2003). Usualmente se las ubica dentro de la superfamilia Palpimanoidea, grupo definido por poseer ciertas características especiales en la morfología de los quelíceros: presencia de setas romas ("peg teeth”) y de la glándula queliceral que se abre en un elevado tubérculo (Forster y Platnick, 1984). No obstante, Schütt (2000, 2002) ha cuestionado la validez de esta agrupación superfamiliar, presentando un esquema alternativo.

En la presente contribución se describen dos nuevas especies para la ciencia, resultado de recientes trabajos de campo llevados a cabo en diversas áreas de bosques valdivianos de Chile y Argentina: Mecysmauchenius platnicki, de Malleco, Chile, y Mecysmauchenioides quetrihue, de Neuquén, Argentina. Esta última es la segunda especie conocida del género mientras que la primera guarda similitudes con Mecysmauchenius canan Forster y Platnick, 1984, de Chiloé.

\section{MATERIAL Y MÉTODOS}

Los especímenes examinados se encuentran depositados en las siguientes instituciones: Museo Nacional de Historia Natural, Santiago (MHNS, Ariel Camousseight) y Museo Argentino de Ciencias Naturales "Bernardino Rivadavia”, Buenos Aires (MACN-Ar, Cristina L. Scioscia). En su mayor parte, las descripciones siguen el formato de Forster \& Platnick (1984). Para aclarar los palpos se los sumergió en aceite esencial de clavo. Se utilizó ocular micrométrico para tomar las mediciones, las cuales se expresan en milímetros.

\section{RESULTADOS}

Mecysmauchenius platnicki, sp. nov.

(Figs. 1-3)

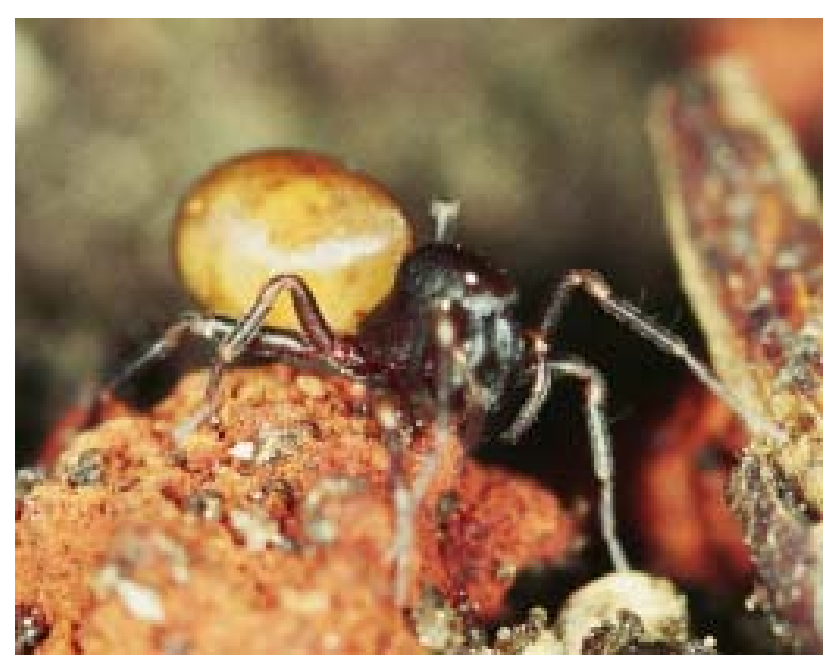

Fig. 1: Mecysmauchenius platnicki, sp. nov., hembra holotipo in vivo.

Tipo: Hembra holotipo de Chile: Región IX (de la Araucanía): Malleco: Monumento Natural Contulmo (Elev. 340m, 3801'S, 73¹1’W), 19-21.XII.1998, en concentrado de hojarasca, M. Ramírez, L. Compagnucci, C. Grismado \& L. Lopardo (fotogramas MJR 4-6; MHNS).

Etimología: El nombre específico es un patronímico en honor al eminente aracnólogo Norman I. Platnick, en reconocimiento a sus numerosos y fundamentales trabajos sobre los diversos grupos de arañas del hemisferio Sur.

Diagnosis: Dentro de las especies “unicolores” del género, la hembra de $M$. platnicki se diferencia de todas, excepto de $M$. canan, por cerecer de la protuberancia subqueliceral (Fig. 2) y de los parches esclerotizados ventrales del abdomen. De $M$. canan se distingue por la amplia escotadura media en el margen anterior del epigastrio (Fig. 3).

Descripción (hembra holotipo): Largo del cefalotórax: 1,72; ancho: 1,00; alto: 1,12. Largo abdomen: 2,08; ancho: 1,70. Coloración (en alcohol): cefalotórax y quelíceros color marrón anaranjado; esternón, labio y enditos del mismo color. Ojos medios posteriores separados entre sí por casi cuatro veces su diámetro transversal, y de los laterales posteriores por casi cinco veces esa medida; altura del clípeo en su punto medio aproximadamente igual a la interdistancia entre los ojos medios posteriores. Area subqueliceral sin protuberancia media (Fig. 2). Largo de los quelíceros: 1,00; ancho: 0,38; superficie posterolateral con un parche de crestas estridulatorias; promargen con dos filas de setas romas; la externa compuesta por nueve pequeñas y la interna por once (más largas progresivamente hacia el ápice), más un grupo de unas trece setas menores dispuestas en torno a la punta de la uña del quelícero. Fémur del palpo con cuatro tubérculos estridulatorios en la superficie prolateral, siendo el proximal el único conspicuo, si bien pequeño; los restantes, minúsculos. Patas marrón anaranjado, más claros en las patelas, tarsos y partes distales de tibias y metatarsos. Longitud de los artejos: fémures, I: 1,06, II: 0,86, III: 0,82, IV: 1,12, palpo: 0,42; patelas, I: 0,36, II: 0,36, III: 0,36, IV: 0,40, palpo: 0,42; tibias, I: 0,82, II: 0,68, III: 0,58, IV: 0,84, palpo: 

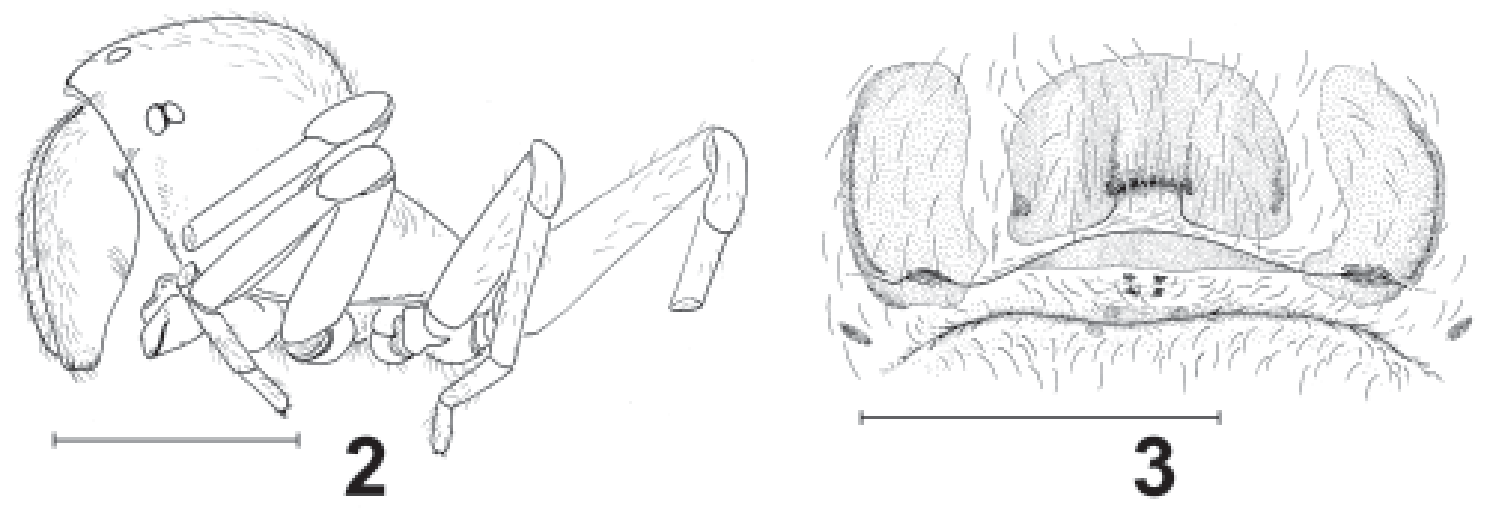

Figs. 2-3: Mecysmauchenius platnicki, sp. nov., hembra holotipo. 2, aspecto lateral del prosoma; 3, epigastrio, vista ventral. Escalas: 0,5 mm.
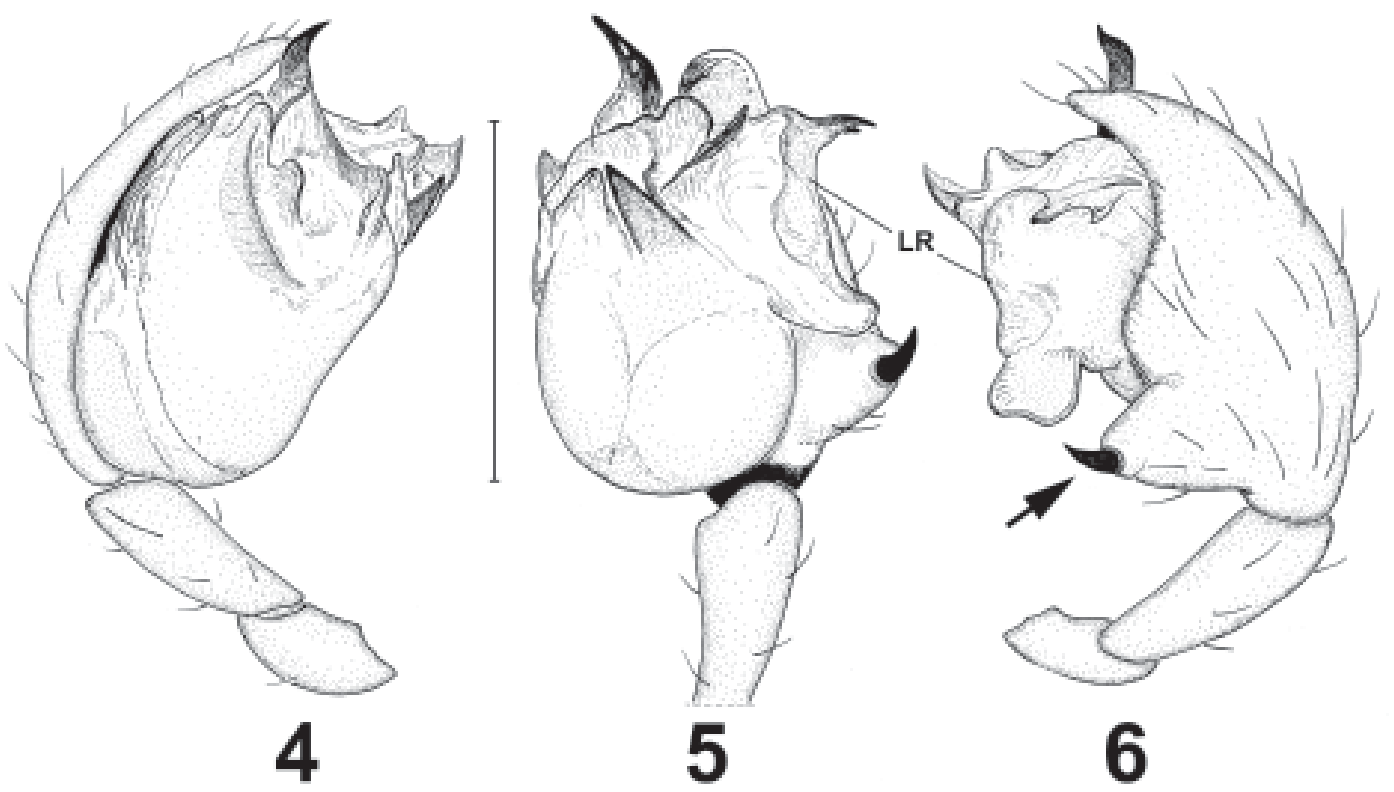

Figs. 4-6: Mecysmauchenioides quetrihue, sp. nov., macho holotipo, palpo izquierdo. 4, prolateral; 5, ventral; 6, retrolateral (LR: lóbulo retrolateral del bulbo; flecha: espina basal del cymbium). Escala: 0,5 $\mathrm{mm}$.

0,44; metatarsos, I: 0,64, II: 0,52, III: 0,52, IV: 0,86; tarsos, I: 0,44, II: 0,34, III: 0,26, IV: 0,32, palpo: 0,52. Abdomen amarillento uniforme; hileras algo más oscuras, amarillentas. Gonoporo con el margen anterior esclerotizado, con una escotadura semicircular; el reborde posterior menos esclerotizado (Fig. 3). Debido a que es el único ejemplar hembra conocido, se prefirió no disecar el abdomen para observar las estructuras genitales internas.

Material examinado: Sólo el holotipo.

Distribución geográfica: Conocida sólo de la localidad típica.
Parentescos: No se pueden proponer afinidad entre esta nueva especie y alguna de las previamente descriptas, debido a que no presenta ningún carácter apomórfico evidente con respecto a sus congéneres. Morfológicamente es muy similar a M. canan, de Chiloé, por ser las únicas con coloración uniforme que carecen de protuberancia subqueliceral y parches abdominales ventrales, pero dichas características no necesariamente relacionan a estas dos especies, pues parecen ser plesiomorfías (Forster y Platnick, 1984).

Mecysmauchenioides quetrihue, sp. nov.

(Figs. 4-6) 
Tipo: Macho holotipo de Argentina: Neuquén: Parque Nacional Nahuel Huapi: península de Quetrihué, 4052' S 713' $\mathrm{W}$, bosque de arrayanes, en hojarasca, 24.II.1996, M. Ramírez (MACN-Ar 10424).

Etimología: El nombre específico es un sustantivo en aposición derivado de la localidad típica.

Diagnosis: Se diferencia de la otra especie del género -M. nordenskjoldi (Tullgren, 1901)-por tener el lóbulo retrolateral del bulbo de forma cóncava, con bordes aplanados y sin dentículos, y por la espina basal del cymbium más corta y menos curvada (Figs. 5-6).

Descripción (macho holotipo): Largo del cefalotórax: 1,66; ancho: 1,04; alto: 1,22. Largo abdomen: 1,68; ancho: 1,24. Coloración (en alcohol): cefalotórax y quelíceros color marrón amarillento; palpos, esternón, labio y enditos más pálidos. Ojos medios posteriores separados entre sí por aproximadamente dos veces y media su diámetro transversal, y de los laterales posteriores por casi tres veces esa medida; altura del clípeo en su punto medio aproximadamente igual a la interdistancia entre los ojos medios posteriores. Largo de los quelíceros: 1,10 ; ancho: 0,34 ; superficie posterolateral con un parche de crestas estridulatorias; promargen con dos filas de setas romas; la externa compuesta por siete pequeñas y la interna por once (más largas progresivamente hacia el ápice), más un grupo de unas diez setas menores dispuestas en torno a la punta de la uña del quelícero. Patas blancuzco amarillento (salvo los fémures, un poco más oscuros); longitud de los artejos: fémures, I: 1,32, II: 1,06, III: 0,90, IV: 1,20, palpo: 0,50; patelas, I: 0,40, II: 0,36, III: 0,34, IV: 0,42, palpo: 0,20; tibias, I: 1,04, II: 0,70, III: 0,60, IV: 0,84, palpo: 0,28; metatarsos, I: 1,00, II: 0,64, III: 0,64, IV: 0,90; tarsos, I: 0,42, II: 0,36, III: 0,26, IV: 0,34, palpo: 0,64. Abdomen blancuzco uniforme, sólo se ven en el dorso -por transparencia- los divertículos entéricos; un área amarillenta por delante de las hileras. Palpo (Figs. 4-6): fémur con un pequeño tubérculo estridulatorio proximal, tibia delgada, sin modificaciones; cymbium con una prolongación basal que lleva una espina basal corta y poco curvada (Figs. 5-6). Bulbo elongado; subtegulum bien visible; complejo grupo de procesos y escleritos de incierta homología (Forster y Platnick, 1984: 31); émbolo no visible, probablemente el ducto eyaculador desemboque en alguno de los procesos lameliformes que se observan en la cara prolateral del bulbo (Fig. 4), si ocurre como en Archaea workmani (Archaeidae, pers. obs.). Los procesos más notorios son el lóbulo retrolateral (probable sinapomorfía del género), que tiene forma cóncava, y la apófisis terminal, curvada y esclerotizada; se destacan también dos procesos laminares aplanados que convergen en la cara ventral del bulbo (Fig. 5).

Hembra: Desconocida.

Material examinado: Mismos datos que el holotipo, un ejemplar inmaduro que presumiblemente pertenece a la misma especie (MACN-Ar 10425).

Distribución geográfica: Conocida sólo de la localidad típica.

\section{AGRADECIMIENTOS}

Deseamos manifestar nuestro agradecimiento a las instituciones y curadores por la posibilidad brindada para el estudio de los especímenes. A Gustavo Hormiga y a dos revisores anónimos por valiosas sugestiones y correcciones sobre el manuscrito. Agradecemos asimismo a la Administración de Parques Nacionales (APN) y a la Corporación Nacional Forestal (CONAF) por la autorización para trabajar en áreas protegidas de Argentina y Chile, respectivamente; a Don Ismael Matamala (guardaparque del Monumento Natural Contulmo) por su colaboración y asistencia, y a Luis Compagnucci y Lara Lopardo (cocolectores en la campaña de Chile) por su inestimable ayuda en el trabajo de campo. La campaña de 1998 en Chile fue financiada por un subsidio de Sigma-Xi para MJR.

\section{REFERENCIAS BIBLIOGRÁFICAS}

CABRERA, A. L. \& WILLINK, A. 1973. Biogeografía de América Latina. Colecc. Monogr. Cient. O.E.A., Biol. 13: 1-122.

FORSTER, R. R. \& PLATNICK, N. I. 1984. A review of the archaeid spiders and their relatives, with notes on the limits of the superfamily Palpimanoidea (Arachnida: Araneae). Bull. Am. Mus. Nat. Hist. 178(1): 1-106.

GRISMADO, C. J. \& LOPARDO, L. 2003. Nuevos datos sobre la distribución geográfica de las familias australes de arañas Malkaridae y Mecysmaucheniidae (Arachnida: Araneae), con la descripción de la hembra de Mecysmauchenius thayerae Forster \& Platnick . Rev. Iber. Aracnol. 8: 37-43.

MORRONE, J. J. 1999. Presentación preliminar de un nuevo esquema biogeográfico de América del Sur. Biogeographica 75(1): 1-16.

MORRONE, J. J. 2000. Biogeographic delimitation of the Subantarctic subregion and its provinces. Rev. Mus. Argentino Cienc. Nat., n. s. 2(1): 1-15.

SCHÜTT, K. 2000. The limits of the Araneoidea (Arachnida: Araneae). Austr. J. Zool. 48(2): 135-153.

SCHÜTT, K. 2002. The limits and phylogeny of the Araneoidea (Arachnida, Araneae). MathematischNaturwissenschaftlichen Fakultät. Berlin, HumboldtUniversität zu Berlin: 153 pp. Disponible en http:// www.dissertation.de (último acceso 8 de octubre de 2004).

Título: Nuevas especies de la familia Mecysmaucheniidae (Araneae) de Chile y Argentina.

Autores: Cristian J. Grismado \& Martín J. Ramírez

Biota Neotropica, Vol. 5( número 1a): 2005

http://www.biotaneotropica.org.br/v5n1a/pt/ abstract?taxonomic-review+bn019051a2005

Recebido em 07/12/2003 - Publicado em 01/02/2005

ISSN 1676-0603 